\title{
Sur les fractions continues monotones non-décroissantes périodiques
}

\author{
Par Folke Ryde
}

Soit donnée une fraction continue de la forme

$$
\frac{a_{1} \mid}{\mid s a_{1}}+\frac{a_{2} \mid}{\mid a_{2}}+\frac{a_{3} \mid}{\mid a_{3}}+\cdots+\frac{a_{m} \mid}{\mid a_{m}}+\cdots,
$$

où $s ; a_{1}, a_{2}, a_{3}, \ldots a_{m}, \ldots$ sont des nombres entiers positifs tels qu'on ait

$$
a_{1} \leqq a_{2} \leqq a_{3} \leqq \cdots \leqq a_{m} \leqq \cdots
$$

Dans un mémoire présenté à l'Académie des sciences de Stockholm ${ }^{1}$, j'ai traité cette classe de fractions continues sous le nom de fractions continues monotones non-décroissantes.

Les fractions continues monotones non-décroissantes sont périodiques si et seulement si tous les nombres $a_{n}$ sont égaux pour tous les indices $n$ à partir d'une certaine valeur $N$. Évidemment chaque fraction continue monotone nondécroissante périodique représente une quantité irrationnelle quadratique réelle. Mais le problème inverse présente des difficultés singulières. Le résultat le plus précis que j'ai obtenu dans cette direction est contenu dans le théorème suivant:

Soit donnée une équation quadratique irréductible $P \theta^{2}+Q \theta+R=0$, dont les coefficients $P, Q$ et $R$ sont des nombres entiers sans aucun facteur commun et dont l'une des racines, soit

satisfait à la condition

$$
\theta=\frac{\sqrt{Q^{2}-4 P R}}{2 P}-\frac{Q}{2 P},
$$

$$
0<\frac{\sqrt{Q^{2}-4 P R}}{2 P}-\frac{Q}{2 P}<1 .
$$

La condition nécessaire et suffisante pour que le développement uniquement déterminé de $\theta$ sous la forme d'une fraction continue monotone non-décroissante soit périodique, c'est-à-dire qu'on ait

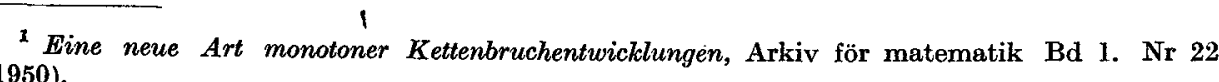
(1950). 
F. RYDE, Sur les fractions continues monotones non-décroissantes périodiques

$$
\begin{aligned}
\frac{\sqrt{Q^{2}-4 P R}}{2 P}-\frac{Q}{2 P}=\frac{a_{1} \mid}{\mid s a_{1}}+ & \frac{a_{2} \mid}{\mid a_{2}}+\frac{a_{3} \mid}{\mid a_{3}}+\cdots+ \\
& +\frac{a_{m-2} \mid}{\mid a_{m-2}}+\frac{a_{m-1} \mid}{\mid a_{m-1}}+\frac{a_{m} \mid}{\mid a_{m}}+\frac{\sigma \mid}{\mid \sigma}+\frac{\sigma \mid}{\mid \sigma}+\cdots
\end{aligned}
$$

ò̀ $s ; a_{1}, a_{2}, a_{3}, \ldots a_{m-2}, a_{m-1}, a_{m}$ et $\sigma$ sont des nombres entiers positifs tels qu'on ait

$$
a_{1} \leqq a_{2} \leqq a_{3} \leqq \cdots \leqq a_{m-2} \leqq a_{m-1} \leqq a_{m} \leqq \sigma
$$

est que

1. $\sigma$ soit contenu dans l'ensemble des nombres entiers

$$
2^{1-n} \cdot\left\{T^{n}+\left(\begin{array}{l}
n \\
2
\end{array}\right) T^{n-2}(T-2)(T+2)+\left(\begin{array}{l}
n \\
4
\end{array}\right) T^{n-4}(T-2)^{2}(T+2)^{2}+\cdots\right\}-2,
$$

où $n$ est un nombre entier positif et où $(T ; U)$ est le système des solutions les plus petites positives de l'équation de Fermat-Pell, $t^{2}-D u^{2}=4$, où $Q^{2}-4 P R=S^{2} D$, $S^{2}$ étant le facteur entier carré le plus grand qui est contenu dans $Q^{2}-4 P R$;

2. $a_{m-1}$ et $a_{m}$ dépendent de $P, Q, R, s, a_{1}, a_{2}, a_{3}, \ldots a_{m-2}$ et $\sigma$ de la manière suivante

$$
a_{m-1}=\frac{\phi \cdot\left(E_{2}-E_{1}\right) \cdot \frac{\sigma}{2} \sqrt{\frac{\overline{Q^{2}-4 P R}}{\sigma(\sigma+4)}-\left\{P E_{1} \phi^{2}+\frac{Q}{2}\left(E_{1}+E_{2}\right) \phi+R E_{2}\right\}}}{E_{1} E_{2}\left(P \phi^{2}+Q \phi+R\right)}
$$

et

$$
a_{m}=\frac{\sigma}{2}-\sqrt{\frac{\sigma(\sigma+4)}{Q^{2}-4 P R}} \cdot \frac{P E_{1} \phi^{2}+\frac{Q}{2}\left(E_{1}+E_{2}\right) \phi+R E_{2}}{\cdot\left(E_{2}-E_{1}\right) \phi},
$$

où

et

$$
\phi=\frac{a_{1} \mid}{\mid s a_{1}}+\frac{a_{2} \mid}{\mid a_{2}}+\frac{a_{3} \mid}{\mid a_{3}}+\cdots+\frac{a_{m-2} \mid}{\mid a_{m-2}}+1
$$

et

$$
E_{1}=1+\frac{1}{\mid a_{m-2}}+\frac{a_{m-2} \mid}{\mid a_{m-3}}+\cdots+\frac{a_{3} \mid}{\mid a_{2}}+\frac{a_{2} \mid}{\mid s a_{1}}
$$

$$
E_{2}=1+\frac{1 \mid}{\mid a_{m-2}}+\frac{a_{m-2} \mid}{\mid a_{m-3}}+\cdots+\frac{a_{3} \mid}{\mid a_{2}} \cdot 1
$$

Dans le cas où $m=2$ les expressions ci-dessus se simplifient en

1 Remarquons ici qu'on peut, en partirant des expressions ci-dessus de $a_{m-1}$ et $a_{m}$, obtenir des expressions de $a_{m}$ et $\sigma$ en $P, Q, R, s, a_{1}, a_{2}, a_{3}, \ldots a_{m-1}$. 
et

$$
a_{1}=\frac{\frac{\sigma}{2} \cdot \sqrt{\frac{Q^{2}-4 P R}{\sigma(\sigma+4)}-\left(\frac{Q}{2}+R s\right)}}{s\left(\frac{P}{s}+Q+R s\right)}
$$

$$
a_{2}=\frac{\sigma}{2}-\sqrt{\frac{\sigma(\sigma+4)}{Q^{2}-4 P R}} \cdot\left(\frac{Q}{2}+R s\right) .
$$

De même, pour $m=1$ les conditions correspondantes deviennent

$$
a_{1}=R \cdot \frac{\frac{\sigma}{Q}}{\frac{2}{2}+R s}=-R \cdot \sqrt{\frac{\sigma(\sigma+4)}{Q^{2}-4 P R}} .
$$

Pour démontrer la nécessité des conditions, supposons que le nombre entier positif $Q^{2}-4 P R$ contienne un facteur entier carré $S^{2}$ et que $S^{2}$ soit le facteur entier carré le plus grand qui est contenu dans $Q^{2}-4 P R$. Posons $Q^{2}-4 P R=$ $=S^{2} D$ et considérons l'équation diophantienne de Fermat-Pell

$$
t^{2}-D u^{2}=4 \text {. }
$$

D'après la théorie générale de cette équation il existe une infinité de solutions en nombres entiers positifs $t$ et $u$, et on les obtient toutes par l'équation

$$
\frac{t+u \sqrt{D}}{2}=\left(\frac{T+U \sqrt{D}}{2}\right)^{n}
$$

où $T, U$ est la solution la plus petite en nombres entiers positifs et où l'exposant doit parcourir tous les nombres entiers positifs. Ainsi nous obtenons

$$
t=2^{\mathrm{i}-n}\left\{T^{n}+\left(\begin{array}{l}
n \\
2
\end{array}\right) T^{n-2} D U^{2}+\left(\begin{array}{l}
n \\
4
\end{array}\right) T^{n-4} \cdot D^{2} U^{4}+\cdots\right\} .
$$

En éliminant $D$ par suite de la relation $D=\left(T^{2}-4\right) / U^{2}$ nous obtenons

$$
\begin{aligned}
t=2^{1-n}\left\{T^{n}+\left(\begin{array}{l}
n \\
2
\end{array}\right) T^{n-2}(T-2)\right. & (T+2)+ \\
& \left.+\left(\begin{array}{l}
n \\
4
\end{array}\right) T^{n-4}(T-2)^{2}(T+2)^{2}+\cdots\right\} .
\end{aligned}
$$

De même, en éliminant $D$ par suite de la relation $D=\left(t^{2}-4\right) / u^{2}$ nous obtenons que le nombre $Q^{2}-4 P R=S^{2} D$ peut s'écrire

$$
Q^{2}-4 P R=S^{2} \cdot D=\frac{S^{2}}{u^{2}}\left(t^{2}-4\right)=\frac{S^{2}}{u^{2}}\left\{(t-2)^{2}+4(t-2)\right\}=\frac{S^{2}}{u^{2}} v(v+4),
$$

où 
F. RYDE, Sur les fractions continues monotones non-décroissantes périodiques

$$
\begin{aligned}
v=t-2=2^{1-n}\left\{T^{n}+\left(\begin{array}{l}
n \\
2
\end{array}\right)\right. & T^{n-2}(T-2)(T+2)+ \\
& \left.+\left(\begin{array}{l}
n \\
4
\end{array}\right) T^{n-4}(T-2)^{2}(T+2)^{2}+\cdots\right\}-2 .
\end{aligned}
$$

Il s'ensuit que le nombre $\theta=\left(\sqrt{Q^{2}-4 P R}-Q\right) / 2 P$ peut s'écrire

$$
\theta=\frac{\sqrt{Q^{2}-4 P R}-Q}{2 P}=\frac{S \sqrt{v(v+4)}-Q u}{2 P u}=\frac{2 S \omega+S v-Q u}{2 P u},
$$

où $\omega=(\sqrt{v(v+4)}-v) / 2$.

Employons maintenant le théorème suivant que j'ai démontré ailleurs: ${ }^{1}$ «Soit donnée une substitution linéaire

$$
\frac{\alpha \omega+\beta}{\gamma \omega+\delta}
$$

dont les coefficients $\alpha, \beta, \gamma$ et $\delta$ sont des nombres entiers quelconques avec la seule restriction que $\alpha \delta-\beta \gamma \neq 0$. Soit aussi donnée une équation quadratique irréductible $p \omega^{2}+q \omega+r=0$, dont les coeffjcients $p, q$ et $r$ sont des nombres entiers sans aucun facteur commun, d'ailleurs quelconques avec la seule restriction qu'entraîne la condition d'irréductibilité. Cela posé, on peut toujours déterminer - et d'une infinité double de manières - une autre substitution linéaire $(A \omega+B) /(C \omega+D)$, dont les coefficients $A, B, C$ et $D$ sont des nombres entiers tels que l'équation quadratique donnée peut s'écrire sous la forme

$$
\frac{\alpha \omega+\beta}{\gamma \omega+\delta}=\frac{A \omega+B}{C \omega+D}
$$

En vertu de ce théorème l'équation $\omega^{2}+v \omega=v$, qui est satisfaite par

$$
\omega=\frac{\sqrt{v^{2}+4 v}-v}{2}
$$

peut s'écrire

$$
\frac{2 S \cdot \omega+S v-Q u}{0 \cdot \omega+2 P u}=\frac{A \omega+B}{C \omega+D}
$$

où $A, B, C$ et $D$ sont des nombres entiers, et, par conséquent, le nombre $\theta=\frac{\sqrt{Q^{2}-4 P R}-Q}{2 P}$ peut toujours s'exprimer et d'ailleurs d'une infinité double de manières sous la forme $\theta=\frac{\sqrt{Q^{2}-4 P R}-Q}{2 P}=\frac{A \omega+B}{C \omega+D}$ et cela pour toute

\footnotetext{
1 Les quantités irrationnelles quadratiques et les substitutions linéaires. Arkiv för matematik. Bd 1. Nr 15 (1949).
} 
valeur donnée de $\omega=\frac{1}{2}(\sqrt{v(v+4)}-v)$, où $v$ est un nombre entier positif arbitrairement choisi parmi l'ensemble des nombres entiers positifs $\left(2^{\prime \prime}\right)$.

Quant aux coefficients $A, B, C$ et $D$ nous obtenons les relations suivantes (3) en identifiant les équations

$$
\begin{gathered}
\frac{2 S \omega+S v-Q u}{2 P u}=\frac{A \omega+B}{C \omega+D} \text { et } \omega^{2}+v \omega=v: \\
\left\{\begin{array}{l}
\frac{C(S v-Q u)-2 P u A+2 S D}{2 S C}=v \\
\frac{D(S v-Q u)-2 P u B}{2 S C}=-v,
\end{array}\right.
\end{gathered}
$$

d'où découle

$$
D=\frac{P u}{S} \cdot A+\frac{S v+Q u}{2 S} \cdot C
$$

et

$$
B=\frac{S v}{P u} \cdot C+\frac{S v-Q u}{2 P u} \cdot D,
$$

d'où par (3A)

$$
\begin{aligned}
B & =\frac{S v}{P u} \cdot C+\frac{S v-Q u}{2 P u}\left(\frac{P u}{S} \cdot A+\frac{S v+Q u}{2 S} \cdot C\right) \\
& =\frac{S v-Q u}{2 S} \cdot A+\left(\frac{S v}{P u}+\frac{S^{2} v^{2}-Q^{2} u^{2}}{4 P u S}\right) \cdot C \\
& =\frac{S v-Q u}{2 S} \cdot A+\frac{S^{2} v(v+4)-Q^{2} u^{2}}{4 P u S} \cdot C \\
& =\frac{S v-Q u}{2 S} \cdot A+\frac{S^{2} D u^{2}-Q^{2} u^{2}}{4 P u S} \cdot C \\
& =\frac{S v-Q u}{2 S} \cdot A+\frac{u}{4 P S}\left(S^{2} D-Q^{2}\right) \cdot C \\
& =\frac{S v-Q u}{2 S} \cdot A-\frac{u}{4 P S} \cdot 4 P R \cdot C
\end{aligned}
$$

et ainsi

$$
B=\frac{S v-Q u}{2 S} \cdot A-\frac{R u}{S} \cdot C
$$


F. RYDE, Sur les fractions continues monotones non-décroissantes pêriodiques

Il s'ensuit que le nombre $\theta=\left(\sqrt{Q^{2}}-4 P \bar{R}-Q\right) / 2 P$ peut toujours s'exprimer sous la forme

$$
\theta=\frac{\sqrt{Q^{2}-4 P R}-Q}{2 P}=\frac{A \omega+B}{C \omega+D}=\frac{A \omega+\frac{S v-Q u}{2 S} \cdot A-\frac{R u}{S} \cdot C}{C \omega+\frac{P u}{S} \cdot A+\frac{S v+Q u}{2 S} \cdot C},
$$

d'où

$$
\theta=\frac{A}{C} \cdot \frac{\omega+\frac{S v-Q u}{2 S}-\frac{R u}{S} \cdot \frac{C}{A}}{\omega+\frac{P u}{S} \cdot \frac{A}{C}+\frac{S v+Q u}{2 S}}
$$

où $\omega=\frac{1}{2}(\sqrt{v(v+4)}-v), \quad v$ étant donné par (2'). L'expression (4) peut être modifiée de la manière suivante. Nous avons $Q^{2}-4 P R=S^{2} D$ et $v(v+4)=D u^{2}$, d'où l'on conclut que

$$
\sqrt{\frac{v(v+4)}{Q^{2}-4 P R}}=\frac{u}{S}
$$

(Remarquons en passant que le nombre $\sqrt{\frac{v(v+4)}{Q^{2}-4 P R}}$ est rationnel.) Nous obtenons

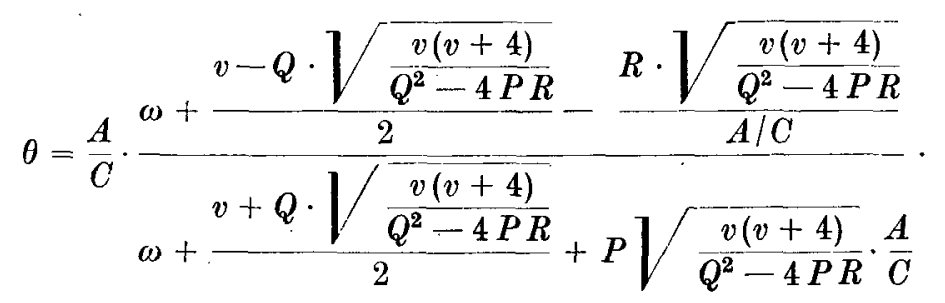

Supposons maintenant pour démontrer la nécessité des conditions du théorème énoncé que le développement de $\theta=\left(\sqrt{Q^{2}-4 P R}-Q\right) / 2 P$ en fraction continue monotone non-décroissante soit périodique, c'est-à-dire de la forme

$$
\begin{aligned}
\theta=\frac{\sqrt{Q^{2}-4 P R}-Q}{2 P}=\frac{a_{1} \mid}{\mid s a_{1}} & +\frac{a_{2} \mid}{\mid a_{2}}+\frac{a_{3} \mid}{\mid a_{3}}+\cdots+ \\
& +\frac{a_{m-2} \mid}{\mid a_{m-2}}+\frac{a_{m-1} \mid}{\mid a_{m-1}}+\frac{a_{m} \mid}{\mid a_{m}}+\frac{\sigma \mid}{\mid \sigma}+\frac{\sigma \mid}{\mid \sigma}+\cdots
\end{aligned}
$$

où $s ; a_{1}, a_{\S}, a_{3}, \ldots . a_{m-2}, a_{m-1}, a_{m}$ et $\sigma$ sont des ortiers positifs tels qu'on ait

$$
a_{1} \leqq a_{2} \leqq a_{3} \leqq \cdots \leqq a_{m-2} \leqq a_{m-1} \leqq a_{m} \leqq \sigma .
$$

D'après la théorie générale des fractions continues nous pouvons écrire 
(5)

$$
\begin{aligned}
\theta=\frac{\sqrt{Q^{2}-4 P R}-Q}{2 P} & =\frac{A_{m}+A_{m-1} \cdot \frac{1}{2}(\sqrt{\sigma(\sigma+4)}-\sigma)}{B_{m}+B_{m-1} \cdot \frac{1}{2}(\sqrt{\sigma(\sigma+4)}-\sigma)} \\
& =\frac{A_{m-1}}{\overline{B_{m-1}}} \cdot \frac{\frac{1}{2}(\sqrt{\sigma(\sigma+4)}-\sigma)+\frac{A_{m}}{A_{m-1}}}{(\sqrt{\sigma(\sigma+4)}-\sigma)+\frac{B_{m}}{B_{m-1}}}
\end{aligned}
$$

où

et

$$
\begin{aligned}
& A_{m} \\
& \dot{B}_{m}
\end{aligned}=\frac{a_{1} \mid}{\mid s a_{1}}+\frac{a_{2} \mid}{\mid a_{2}}+\frac{a_{3} \mid}{\mid a_{3}}+\cdots+\frac{a_{m-2} \mid}{\mid a_{m-2}}+\frac{a_{m-1} \mid}{\mid a_{m-1}}+\frac{a_{m} \mid}{\mid a_{m}}
$$

$$
\frac{A_{m-1}}{B_{m-1}}=\frac{a_{1} \mid}{\mid s a_{1}}+\frac{a_{2} \mid}{\mid a_{2}}+\frac{a_{3} \mid}{\mid a_{3}}+\cdots+\frac{a_{m-2} \mid}{\mid a_{m-2}}+\frac{a_{m-1} \mid}{\mid a_{m-1}},
$$

car la quantité positive

$$
x=\frac{\sigma \mid}{\mid \sigma}+\frac{\sigma \mid}{\mid \sigma}+\cdots
$$

satisfait évidemment à l'équation quadratique $x^{2}+\sigma x=\sigma$.

En identifiant les expressions $\left(4^{\prime}\right)$ et $(5)$ de $\theta$ nous obtenons

(6) $\frac{A_{m-1}}{B_{m-1}} \cdot \frac{\sqrt{\sigma(\sigma+4)}-\sigma+\frac{2 A_{m}}{A_{m-1}}}{\sqrt{\sigma(\sigma+4)}-\sigma+\frac{2 B_{m}}{B_{m-1}}}=$

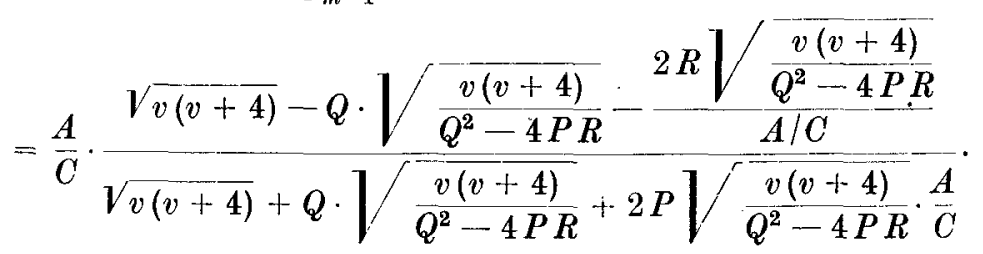

En posant

$$
\begin{aligned}
K & =\frac{A_{m-1}}{B_{m-1}} \\
k & =\frac{A}{C} \\
M & =-\sigma+\frac{2 A_{m}}{A_{m-1}} \\
N & =-\sigma+\frac{2 B_{m}}{B_{m-1}} \quad(M \neq N)
\end{aligned}
$$


F. RYDE, Sur les fractions continues monotones non-décroissantes périodiques

$$
\begin{aligned}
& M_{1}=-\left(Q+2 R \cdot \frac{C}{A}\right) \sqrt{\frac{v(v+4)}{Q^{2}-4 P R}} . \\
& \left.N_{1}=\left(Q+2 P \cdot \frac{A}{C}\right)\right] \sqrt{\frac{v(v+4)}{Q^{2}-4 P R}} \quad\left(M_{1} \neq N_{1}\right),
\end{aligned}
$$

où toutes les expressions sont rationnelles, l'équation (6) se simplifie en

d'où découle

$$
K \cdot \frac{\sqrt{\sigma(\sigma+4)}+M}{\sqrt{\sigma(\sigma+4)}+N}=k \cdot \frac{\sqrt{v(v+4)}+M_{1}}{\sqrt{v(v+4)}+N_{1}}
$$

et par suite

$$
K+K \cdot \frac{M-N}{\sqrt{\sigma(\sigma+4)}+N}=k+k \cdot \frac{M_{1}-N_{1}}{\sqrt{v(v+4)}+N_{1}}
$$

$$
K+\frac{K(M-N)(\sqrt{\sigma(\sigma+4)}-N)}{\sigma(\sigma+4)-N^{2}}=k+\frac{k\left(M_{1}-N_{1}\right)\left(\sqrt{v(v+4)}-N_{1}\right)}{v(v+4)-N_{1}^{2}} .
$$

On en déduit

$$
\begin{aligned}
& \frac{K(M-N)}{\sigma(\sigma+4)-N^{2}} \cdot \sqrt{\sigma(\sigma-4)}-\frac{k\left(M_{1}-N_{1}\right)}{v(v+4)-N_{1}^{2}} \cdot \sqrt{v(v+4)}= \\
& =k-K+\frac{K N(M-N)}{\sigma(\sigma+4)-N^{2}}-\frac{k N_{1}\left(M_{1}-N_{1}\right)}{v(v+4)-N_{1}^{2}} .
\end{aligned}
$$

En observant que l'expression à droite est rationnelle tandis que l'expression à gauche est irrationnelle, à moins qu'elle ne soit nulle, nous obtenons que le nombre $\frac{\sqrt{\sigma(\sigma+4)}}{\sqrt{v(v+4)}}$ doit être rationnel, soit $=\frac{p}{q}$, où $p$ et $q$ sont des nombres entiers positifs sans aucun facteur commun. Cela posé, nous obtenons

$$
\sigma(\sigma+4)=(\sigma+2)^{2}-4=\frac{p^{2}}{q^{2}} \cdot\left((v+2)^{2}-4\right) .
$$

Mais $(v+2, u)$ est une solution de l'équation $t^{2}-4=D u^{2}$. Il s'ensuit que

$$
(\sigma+2)^{2}-4=\frac{p^{2} u^{2}}{q^{2}} \cdot D
$$

En observant que l'expression à gauche représente un nombre entier, nous obtenons que $\frac{p u}{q}$ doit être aussi un nombre entier. En effet, nous avons supposé que le nombre $D$ ne contienne pas de facteur carré. Par conséquent, $q$ ne peut pas contenir de facteur qui n'entre pas comme facteur en $u$, car au cas con- 
traire $D$ doit contenir ce facteur doublement, ce qui est impossible. Il s'ensuit que $(\sigma+2, p u / q)$ est une solution de l'équation (1) de Fermat-Pell et, par conséquent, $\sigma$ est contenu dans l'ensemble des nombres entiers (2").

Nous pouvons donc fixer la valeur du nombre $v$ en le posant égal à $\sigma$. Observons de plus que les nombres entiers $A$ et $C$ dans l'expression (4') sont encore indéterminés. Ainsi nous sommes en droit de fixer leurs valeurs de la manière suivante $A=2 S A_{m-1}, C=2 S B_{m-1}$, d'où

$$
\frac{A}{C}=\frac{A_{m-1}}{B_{m-1}}
$$

En abrégeant nous posons $\frac{A}{C}=\frac{A_{m-1}}{B_{m-1}^{-}}=\phi$. Alors l'équation (6) peut s'écrire

$$
\frac{\sqrt{\sigma(\sigma+4)}-\sigma+\frac{2 A_{m}}{A_{m-1}}}{\sqrt{\sigma(\sigma+4)}-\sigma+\frac{2 B_{m}}{B_{m-1}}}=\frac{\sqrt{\sigma(\sigma+4)}-\left(Q+\frac{2 R}{\phi}\right) r}{\sqrt{\sigma(\sigma+4)}+(Q+2 P \phi) r},
$$

où $r=\sqrt{\frac{\sigma(\sigma+4)}{Q^{2}-4 P R}}$ désigne un nombre rationnel. De cela découle

$$
\begin{aligned}
\sqrt{\sigma(\sigma+4)} \cdot\left(r(Q+2 P \phi)+\frac{2 A_{m}}{A_{m-1}}-\frac{2 B_{m}}{B_{m-1}}+r\left(Q+\frac{2 R}{\phi}\right)\right) \\
=r\left(\sigma-\frac{2 A_{m}}{A_{m-1}}\right)(Q+2 P \phi)+r\left(\sigma-\frac{2 B_{m}}{B_{m-1}}\right)\left(Q+\frac{2 R}{\phi}\right) .
\end{aligned}
$$

En observant que l'expression à droite est rationnelle tandis que l'expression à gauche est irrationnelle, à moins que la parenthèse dans l'expression à gauche ne soit nulle, nous obtenons

$$
\frac{B_{m}}{B_{m-1}}-\frac{A_{m}}{A_{m-1}}=\frac{r}{\phi} \cdot\left(P_{\phi^{2}}+Q_{\phi}+R\right)
$$

et

$$
\frac{A_{m}}{A_{m-1}} \cdot(Q+2 P \phi)+\frac{B_{m}}{B_{m-1}} \cdot\left(Q+\frac{2 R}{\phi}\right)=\frac{\sigma}{\phi} \cdot\left(P \phi^{2}+Q \phi+R\right) .
$$

Remarquons en passant qu'il découle de (8) et (9) en éliminant $\left(P \phi^{2}+Q_{\phi}+R\right) / \phi$ que

$$
\frac{A_{m}}{\bar{B}_{m}}=\frac{\sigma \phi-Q r \phi-2 R r}{\sigma+Q r+2 \operatorname{Pr} \phi} .
$$

D'après la théorie générale des fractions continues nous avons (cf. 0 . Perron, Die Lehre von den Kettenbrüchen, Leipzig und Berlin 1929, p. 12) 
F. RYDE, Sur les fractions continues monotones non-décroissantes périodiques

où

$$
\frac{B_{m}}{B_{m-1}}=a_{m}+\frac{a_{m} \mid}{\mid a_{m-1}}+\frac{a_{m-1} \mid}{\mid a_{m-2}}+\cdots+\frac{a_{3} \mid}{\mid a_{2}}+\frac{a_{2} \mid}{\mid s a_{1}}=a_{m}+\frac{a_{m}}{a_{m-1} \cdot E_{1}},
$$

$$
E_{1}=1+\frac{1 \mid}{\mid a_{m-2}}+\frac{a_{m-2} \mid}{\mid a_{m-3}}+\cdots+\frac{a_{3} \mid}{\mid a_{2}}+\frac{a_{2} \mid}{\mid s a_{1}} \cdot(m>2) .
$$

De même nous posons

où

$$
\frac{A_{m}}{A_{m-1}}=a_{m}+\frac{a_{m} \mid}{\mid a_{m-1}}+\frac{a_{m-1} \mid}{\mid a_{m-2}}+\cdots+\frac{a_{4} \mid}{\mid a_{3}}+\frac{a_{3} \mid}{\mid a_{2}}=a_{m}+\frac{a_{m}}{a_{m-1} \cdot E_{2}},
$$

$$
E_{2}=1+\frac{1}{\mid a_{m-2}}+\frac{a_{m-2} \mid}{\mid a_{m-3}}+\cdots+\frac{a_{4} \mid}{\mid a_{3}}+\frac{a_{3} \mid}{\mid a_{2}} \cdot(m>2) \cdot\left(E_{2}=1 \text { pour } m=3\right) .
$$

En faisant usage de ces relations on trouve par l'intermédiaire de (8)

d'où découle

$$
\frac{a_{m}}{a_{m-1} \cdot E_{1}}-\frac{a_{m}}{a_{m-1} \cdot E_{2}}=\frac{r}{\phi} \cdot\left(P \phi^{2}+Q \phi+R\right),
$$

$$
\frac{a_{m}}{a_{m-1}}=\frac{E_{1} E_{2}}{E_{2}-E_{1}} \cdot \frac{r}{\phi} \cdot\left(P \phi^{2}+Q \phi+R\right)
$$

et par l'intermédiaire de (9)

$$
\begin{aligned}
\left(a_{m}+\frac{a_{m}}{a_{m-1}} \cdot \frac{1}{E_{2}}\right)(Q+2 P \phi) & +\left(a_{m}+\frac{a_{m}}{a_{m-1}} \cdot \frac{1}{E_{1}}\right)\left(Q+\frac{2 R}{\phi}\right) \\
& =\frac{\sigma}{\phi} \cdot\left(P \phi^{2}+Q \phi+R\right),
\end{aligned}
$$

d'où découle

$$
2 a_{m}\left(Q+P_{\phi}+\frac{R}{\phi}\right)=\frac{\sigma}{\phi} \cdot\left(P \phi^{2}+Q \phi+R\right)-\frac{a_{m}}{a_{m-1}} \cdot\left(\frac{Q+2 P \phi}{E_{2}}+\frac{Q+\frac{2 R}{\phi}}{E_{1}}\right) .
$$

En remplaçant ici $a_{m} / a_{m-1}$ par l'expression dans $\left(8^{\prime}\right)$ nous obtenons

$$
\begin{aligned}
& \frac{2 a_{m}}{\phi} \cdot\left(P \phi^{2}+Q \phi+R\right)=\frac{\sigma}{\phi} \cdot\left(P \phi^{2}+Q \phi+R\right)- \\
& \quad-\frac{E_{1} E_{2}}{E_{2}-E_{1}} \cdot \frac{r}{\phi} \cdot\left(P \phi^{2}+Q_{\phi}+R\right) \cdot\left(\frac{Q+2 P \phi}{E_{2}}+\frac{Q+\frac{2 R}{\phi}}{E_{1}}\right) .
\end{aligned}
$$

En divisant par l'expression $2\left(P \phi^{2}+Q_{\phi}+R\right) / \phi$, qui ne peut pas s'annuler, on trouve, en tenant compte de la valeur de $r$, 


$$
a_{m}=\frac{\sigma}{2}-\sqrt{\frac{\sigma(\sigma+4)}{Q^{2}-4 P R}} \cdot \frac{P E_{1} \phi^{2}+Q \cdot \frac{E_{1}+E_{2}}{2} \phi+R E_{2}}{\left(E_{2}-E_{1}\right) \phi} \text {. }
$$

Par l'intermédiaire de $\left(8^{\prime}\right)$ nous obtenons finalement

$$
a_{m-1}=\frac{\phi \cdot\left(E_{2}-E_{1}\right) \cdot \frac{\sigma}{2} \sqrt{\frac{Q^{2}-4 P \bar{R}}{\sigma(\sigma+4)}-\left(P E_{1} \phi^{2}+Q \cdot \frac{E_{1}+E_{2}}{2} \phi+R E_{2}\right)}}{E_{1} E_{2} \cdot\left(P \phi^{2}+Q \phi+R\right)} .
$$

Il s'ensuit que lés conditions du théorème énoncé sont de nécessité remplies.

Il reste à démontrer que les conditions du théorème en question sont suffisantes pour que le nombre irrationnel

$$
\theta=\frac{a_{1} \mid}{\mid s a_{1}}+\frac{a_{2} \mid}{\mid a_{2}}+\frac{a_{3} \mid}{\mid a_{3}}+\cdots+\frac{a_{m-2} \mid}{\mid a_{m-2}}+\frac{a_{m-1} \mid}{\mid a_{m-1}}+\frac{a_{m} \mid}{\mid a_{m}}+\frac{\sigma \mid}{\mid \sigma}+\frac{\sigma \mid}{\mid \sigma}+\cdots,
$$

où $s ; a_{1}, a_{2}, a_{3}, \ldots a_{m-2}, a_{m-1}, a_{m}, \sigma$ sont des nombres entiers positifs tels qu'on ait

$$
a_{1} \leqq a_{2} \leqq a_{3} \leqq \cdots \leqq a_{m-2} \leqq a_{m-1} \leqq a_{m} \leqq \sigma,
$$

satisfasse à l'équation quadratique $P \theta^{2}+Q \theta+R=0$. D'après la théorie générale des fractions continues nous pouvons écrire (cf. (5))

$$
\begin{gathered}
\theta=\frac{A_{m-1} \cdot \varphi+A_{m}}{B_{m-1} \cdot \varphi+B_{m}}=\frac{A_{m-1}}{B_{m-1}} \cdot \frac{\varphi+A_{m} / A_{m-1}}{\varphi+B_{m} / B_{m-1}} \\
=\phi \cdot \frac{\varphi+a_{m}+\frac{a_{m}}{a_{m-1} \cdot E_{2}}}{\varphi+a_{m}+\frac{a_{m}}{a_{m-1} \cdot E_{1}}}
\end{gathered}
$$

selon la définition de $\phi, E_{1}$ et $E_{2}$ et en posant

$$
\varphi=\frac{\sqrt{\sigma(\sigma+4)}-\sigma}{2}=\frac{\sigma \mid}{\mid \sigma}+\frac{\sigma \mid}{\mid \sigma}+\cdots
$$

En divisant les expressions de $a_{m}$ et $a_{m-1}$ de l'énoncé du théorème en question on obtient

$$
\frac{a_{m}}{a_{m-1}}=\frac{E_{1} E_{2}}{E_{2}-E_{1}} \cdot \sqrt{\frac{\sigma(\sigma+4)}{Q^{2}-4 P R}} \cdot \frac{P \phi^{2}+Q \phi+R}{\phi} .
$$

En substituant aussi les expressions de $\varphi$ et de $a_{m}$ nous obtenons 
F. RYDE, Sur les fractions continues monotones non-décroissantes périodiques

$$
\begin{aligned}
& \theta=\phi \cdot \frac{\frac{\sqrt{\sigma(\sigma+4)}}{2}-\sqrt{\frac{\sigma(\sigma+4)}{Q^{2}-4 P R} \cdot \frac{P E_{1} \phi+\frac{Q}{2}\left(E_{1}+E_{2}\right)+\frac{R E_{2}}{\phi}}{E_{2}-E_{1}}}}{\frac{\sqrt{\sigma(\sigma+4)}}{2}-\sqrt{\frac{\sigma(\sigma+4)}{Q^{2}-4 P R} \cdot \frac{P E_{1} \phi+\frac{Q}{2}\left(E_{1}+E_{2}\right)+\frac{R E_{2}}{\phi}}{E_{2}-E_{1}}}+} \\
& \frac{+\frac{E_{1}}{E_{2}-E_{1}} \sqrt{\frac{\sigma(\sigma+4)}{Q^{2}-4 P R} \cdot \frac{P \phi^{2}+Q \phi+R}{\phi}}}{+\frac{E_{2}}{E_{2}-E_{1}} \sqrt{\frac{\sigma(\sigma+4)}{Q^{2}-4 P R}} \cdot \frac{P \phi^{2}+Q \phi+R}{\phi}},
\end{aligned}
$$

d'où

$$
\begin{aligned}
& \theta=\phi \frac{\frac{\sqrt{Q^{2}-4} \overline{P R}}{2}+\frac{1}{E_{2}-E_{1}} \cdot\left(Q E_{1}+\frac{R E_{1}}{\phi}-\frac{Q E_{1}}{2}-\frac{Q E_{2}}{2}-\frac{R E_{2}}{\phi}\right)}{\frac{\sqrt{Q^{2}-4 P R}}{2}+\frac{1}{E_{2}-E_{1}} \cdot\left(P E_{2} \phi+Q E_{2}-P E_{1} \phi-\frac{Q E_{1}}{2}-\frac{Q E_{2}}{2}\right)} \\
& =\phi \cdot \frac{\frac{\sqrt{Q^{2}-4} \overline{P R}}{2}-\frac{Q}{2}-\frac{R}{\phi}}{\frac{\sqrt{Q^{2}-4}}{2}+\frac{Q}{2}+P \phi}
\end{aligned}
$$

En multipliant le numérateur ainsi que le dénominateur par $\left(\sqrt{Q^{2}-4 P R}-Q\right) / 2$ nous obtenons

$$
\begin{aligned}
\theta=\phi & \frac{\left(\frac{\sqrt{Q^{2}-4 P R}}{2}-\frac{Q}{2}-\frac{R}{\phi}\right)\left(\frac{\left.\sqrt{Q^{2}-4 P R}-\frac{Q}{2}\right)}{2}-4 P R\right.}{4}-\frac{Q^{2}}{4}+P_{\phi}\left(\frac{\sqrt{Q^{2}-4 P R}}{2}-\frac{Q}{2}\right) \\
=\phi & \frac{\left(\frac{\sqrt{Q^{2}-4 P R}}{2}-\frac{Q}{2}-\frac{R}{\phi}\right)\left(\frac{\sqrt{Q^{2}-4 P R}}{2}-\frac{Q}{2}\right)}{P_{\phi}\left(\frac{\sqrt{Q^{2}-4 P R}}{2}-\frac{Q}{2}-\frac{R}{\phi}\right)}=\frac{\sqrt{Q^{2}-4 P R}}{2 P}-\frac{Q}{2 P},
\end{aligned}
$$

c'est-à-dire, le nombre $\theta$ satisfait à l'équation $P \theta^{2}+Q \theta+R=0$. 